\title{
LncRNA BANCR Facilitates Melanoma Cells Growth, Migration and Invasion by Inhibiting miR-206 and miR-571 to Induce G6PD Expression
}

\section{Yuye Yang}

Kunming Medical University

Huixin Yang

Kunming Medical University

Zihan Yi

Kunming Medical University

Lijuan Yang

Kunming Medical University

Yueli Ni

Kunming Medical University

\section{Meiling Zhou}

Kunming Medical University

\section{Guohang Xiong}

Kunming Medical University

Yannick Luther AGBANA

Kunming Medical University

Lei Zhao

Kunming Medical University

Zhe Yang

Kunming Medical University

Qiao Zhang

Kunming Medical University

\section{Yingmin Kuang}

Kunming Medical University

Yuechun Zhu ( $\square$ Zhuyuechun180522@163.com )

Kunming Medical University

\section{Research article}

Keywords: BANCR, G6PD, miR-206, miR-571, Melanoma 
Posted Date: June 29th, 2020

DOl: https://doi.org/10.21203/rs.3.rs-31582/v1

License: (c) (i) This work is licensed under a Creative Commons Attribution 4.0 International License. Read Full License 


\section{Abstract}

Background Here, we evaluated the roles of LncRNA BANCR in facilitating melanoma cells growth, migration, invasion. Moreover, we examined the molecular mechanisms of BANCR in promoting melanoma development.

Methods Here, we measured BANCR expression by quantitative PCR. By transwell assay, we detected the cancer cell migration and invasion. The melanoma growth and migration induced by BANCR was detected by colony formation assay and in xenograft nude mice model. By Western blotting and luciferase assay, we evaluated the molecular mechanism of BANCR in inhibiting miR-206 and miR-571 to induce G6PD expression.

Results We found BANCR was overexpressed in melanoma cells and tissues. In addition, we showed that BANCR promoted melanoma cells growth in vitro and in vivo. Moreover, BANCR promotes cancer cell migration and invasion in vitro, and induced melanoma metastasis in nude mice model. The migration and invasion induced by BANCR in melanoma cells were involved in upregulation of matrix metalloproteinases (MMPs) MMP2 and MMP9. Mechanismly, we indicated BANCR promoted melanoma cell growth and migration by suppressing miR-206 and miR-571 expression to activate glucose metabolism pathway and induce G6PD expression. Furthermore, miR-206 and miR-571 inhibited G6PD expression by directly binding the 3'UTR of G6PD. We also showed miR-206 and miR-571 inhibited the proliferation of melanoma cells.

Conclusion Our findings indicated that BANCR contributed to melanoma development, which might provide novel insights into the function of IncRNA-driven carcinogenesis in melanoma.

\section{Background}

Melanoma is a rare and serious skin cancer with high metastatic rate and poor prognosis [1]. The major risk factor for melanoma is ultraviolet. Melanoma at early stage can be treated by surgery, but surgery treatment for late stage melanoma is difficult and inefficient [2]. Thus, it is very important to diagnose melanoma at early stage and develop efficient molecular targeted drugs for melanoma. Currently, the mechanisms driving melanoma tumorigenesis and metastasis remains unclear. Thus, it is critical to clarify the biological mechanisms underlying melanoma progression for the development of new and effective strategies for melanoma treatment.

Long noncoding RNAs (IncRNAs) are a novel class of RNAs with more than 200 nucleotides in length with limited protein coding capacity [3]. LncRNAs exert very important roles in many biological functions such as cell growth, proliferation, differentiation, metastasis, invasion, apoptosis and migration [4-6]. Moreover, multiple studies report that IncRNAs regulate complex and diverse biological functions, such as embryonic stem cell pluripotency [7]. Notably, many IncRNAs are showed to be deregulated in several cancer types including bladder, lung, breast, gastric cancer and glioma [4]. LncRNAs with miRNA response elements (MREs) may exert competing endogenous RNA (ceRNA) roles to act as endogenous decoys for 
miRNAs. LncRNAs promote cancer growth by participating in ceRNA networks and crosstalk between mRNA and miRNA [3].

LncRNA BANCR (BRAF-activated non-protein coding RNA) with 693-nucleotide lenth is transcribed from human chromosome 9 [8]. BANCR has been reported to promote cell proliferation, migration and epithelial-mesenchymal transition (EMT) in melanoma and other cancer [9-11]. For example, BANCR is overexpressed in retinoblastoma, and depletion of BANCR decreases cancer cell progress in retinoblastoma [12]. Although several studies show that BANCR display an oncogene property, others studies report BANCR as a tumor suppressor in lung cancer and colon cancer [13]. Understanding the function and mechanism of BANCR in tumor is just beginning.

\section{Methods}

\section{Cell line}

Cell lines A375, A875, sk-mel-110, M21, sk-mel-28 (M28) and Hacat were obtained from the Cell Bank of Type Culture Collection of the Chinese Academy of Sciences (Shanghai, China). Cells were cultured in high-glucose Dulbecco's modified Eagle's medium (Invitrogen, Grand Island, NY, USA) with 10\% fetal bovine serum (Hyclone Laboratories, Inc., South Logan, UT, USA) in a humidified atmosphere containing $5 \% \mathrm{CO} 2$ at $37^{\circ} \mathrm{C}[14]$.

\section{Clinical samples}

Forty-eight melanoma tumor tissues and the corresponding adjacent normal samples were obtained from the Department of orthopedics of Yunnan Cancer Hospital in Kunming. Informed consent was obtained from all patients. These 48 patients underwent surgery between 2014 and 2016.

\section{Extraction of RNAand PCR}

Total RNA from tissue or cells was extracted using TRIzol reagent (Invitrogen, Grand Island, NY, USA), according to the manufacturer's instructions. qRT-PCR was performed by using SYBR Green (TransGen Biotech) according to instructions in the user's manual. The data were acquired from an $A B I 7500$ realtime PCR instrument (Applied Biosystems, Foster City, CA, USA). The $2^{-\triangle \triangle \mathrm{Ct}}$ method was used to measure the mRNA levels [15].

For the primers, G6PD: 5-TCATCATCATGGGTGCATCGG-3 (sense), 5- CTTGAAGAAGGGCTCACTCTGTTTG3 (antisense); BANCR: 5- CTCGCTTTCACTTTATGGATTC-3 (sense), 5-GGTCAGGGGTCTCTTCAG-3 (antisense); MiR-206: 5-CCGTGGAATGTAAGGAAGTGTGTGG-3 (forward), MiR-571: 5CGTGAGTTGGCCATCTGAGTGAG-3 (forward); Reverse-primer (Uni-miR RT-PCR primer, included in SYBR Premix Ex Taq kit (TaKaRa, Japan)). U6: 5-CTCGCTTCGGCAGCACA-3 (forward), 5AACGCTTCACGAATTTGCGT-3 (reverse). 
For cell viability analysis, MTT assay was conducted to assess the cell viability. The MTT experiments were performed as previously described. Clonogenic cell survival experiments were performed as previously described [16]. These assays were conducted in triplicate.

\section{Cell cycle analysis}

Cells were collected and fixed with $70 \%$ ethanol in a fixative at $-20^{\circ} \mathrm{C}$ overnight. Cells were then stained with propidium iodide (Invitrogen, Grand Island, NY, USA) and detected by flow cytometry (BD

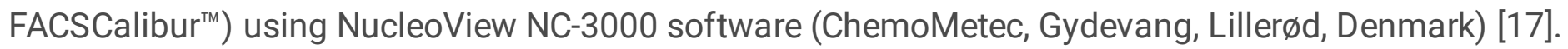

\section{Western blotting}

This assay was performed as previously described [18]. Protein (40-100 ug) were separated by sodium dodecyl sulfate polyacrylamide gel electrophoresis in 8-15\% resolving gel using a Mighty Small II Deluxe Mini Vertical Electrophoresis Unit (Hoefer, Inc., Holliston, MA, USA). Proteins were then electrotransferred to PVDF membranes. Subsequently, the PVDF membranes were blocked with a blocking buffer ( $5 \%$ nonfat dry milk or $5 \%$ BSA in TBST buffer) for $1-2 \mathrm{~h}$ at room temperature. The membranes were incubated with the antibodies overnight at $4{ }^{\circ} \mathrm{C}$. The MMP2, MMP9 and G6PD antibodies were acquired from CST, Inc., Beverly, MA, USA), GAPDH (Santa Cruz, Inc. Santa Cruz, CA, USA). The next day, blots were incubated with a horseradish peroxidase-conjugated secondary antibody. The signals were stimulated with Enhanced Chemiluminescence Substrate (GE Healthcare; Munich, Germany) and visualized on Image Quant 4000.

\section{Cell migration and invasion assay}

Cell migration and invasion experiments were performed as previously described [17].

\section{Luciferase assay}

For generation of promoter reporters, the human $B A N C R$ regions were PCR amplified from genomic DNA of $A 375$ cells with the following primers. BANCR Forword primers:

5'ccgCTCGAGATTCCCTTACTTTCTTAATAAAC3', Reverse primers:

5'ataagaatGCGGCCGCTTAGGATTTTTTAAATTTTATTTTTATTTTTTTTTTTAGAAAAGAG3'; mutBANCR Forword primers: 5'CCAATGGTAACCAGGTGGGGCGATCATCTTGCCCAGAAACATC3', Reverse primers: 5'CAAGATGATCGCCCCACCTGGTTACCATTGGTGCTGCAGTCTATTTCC3'; mutBANCR-206 Forword primers: 5'CATTGTGATGGCACCTCCTCTCTTTTCTAAA3'; Reverse primers:

5'AAGAGAGGAGGTGCCATCACAATGAAGAAAGCCTG 3'; G6PD Forword primers:

5'ACGCGTCGACGCCCTGGGCACCCACCTCCACC 3', Reverse primers

5'ATAAGAATGCGGCCGCTTGGGCTGTTTGCGGATTTAATG 3'

G6PD and phosphofructokinase (PFK) activity assay 
To measure G6PD and PFK activity activity, the G6PD and PFK activity detect kits (Jiemei Bio, shanghai, China) were used according to the manufacturer's instructions.

\section{Animal studies}

For subcutaneous tumorigenicity assay, four-week-old BALB/c nude mice were purchased from the National Laboratory Animal Center (Shanghai, China). The animal studies were approved by the Institutional Animal Care and Use Committee of Kunming Hospital. Great efforts were made to minimize the number and suffering of animals. A375 cells $\left(2 \times 10^{6}\right)$ were re-suspended in growth media $(150 \mu \mathrm{L})$ and Matrigel substrate $(150 \mu \mathrm{L})$. And then these cells were injected in nude mice. The animals were euthanized via overdose of pentobarbital $(100 \mathrm{mg} / \mathrm{kg})$ at 33 days, and the tumors were collected to measure their weights and volumes. Tumor volume was measured and calculated with the formula: volume $\left(\mathrm{mm}^{3}\right)=$ length $\times$ width $^{2} / 2$ [19]. For lung metastases model, each mouse was injected with $1 \times 10^{6}$ cells in 200 ul PBS by tail vein injection. After 4 weeks post-injection, lung metastases were monitored and quantified. The animals were euthanized via overdose of pentobarbital $(100 \mathrm{mg} / \mathrm{kg})$ at 33 days, the tumors and lungs were excised. These tissues were fixed in formalin and embedded in paraffin. HE staining assay were performed by pathologists.

\section{Statistical analysis}

Data analyses were performed using SPSS 20.0 software (SPSS, Inc., Chicago, IL, USA). The data were analysed using Student's t-test. All data were displayed as the mean \pm SD. $P$ values $<0.05$ were considered statistically significant.

\section{Results}

\section{LncRNA BANCR is overexpressed in melanoma and promotes cell growth in vitro and in vitro}

To examine LncRNA BANCR was involved in melanoma, we analyzed LncRNA BANCR levels in 48 malignant human melanoma sample and adjacent normal tissue by RT-PCR. We found the BANCR levels were significantly increased in melanoma tissues relative to adjacent normal tissues (Fig. 1a). To assess the biological role of BANCR in melanoma cells, we constructed stably overexpressed BANCR melanoma cells (A375-BANCR OE) by BANCR overexpression vector lenti-viral systems and knockdown of BANCR cells (A375-BANCR KD) by shRNA lenti-viral systems. The expression levels of BANCR in A375-BANCR OE and A375-BANCR KD cells were also determined by RT-PCR. The results revealed that BANCR levels were significantly increased in A375-BANCR OE cells compared with control cells (A375-LV5), whereas BANCR levels were significantly decreased in A375-BANCR KD cells compared with control cells (A375-GL2) (Fig. 1b). By cell viability assay, we showed that upregulation of BANCR obviously promoted cell proliferation in A375 cells. By contrast, downregulation of BANCR clearly inhibited cell proliferation in A375 cells (Fig. 1c). In addition, by colony formation assay, we showed that knockdown of BANCR inhibited A375 cells colony formation, whereas overexpression of BANCR promoted A375 cells colony formation (Fig. 1d). Moreover, to assess the role of BANCR in the regulation of cell cycle in A375 cells, we performed cell cycle 
analysis by flow cytometry. As shown in Fig. 1e, the G0/G1 phase cells proportion was significantly increased in A375-BANCR KD cells. However, in A375-BANCR OE cells, the G0/G1 phase cells proportion was significantly decreased. Based on these results, we further investigated the expression of cell cycleassociation proteins CDK2 and CyclinD1 in A375-BANCR KD and A375-BANCR OE cells. The results indicated knockdown of BANCR downregulated the CDK2 and CyclinD1 levels, whereas overexpression of BANCR enhanced the two proteins levels in A375 cells (Fig. 1f). To further determine whether BANCR affected cell proliferation of melanoma in vivo, A375-BANCR KD and A375-BANCR OE cells were inoculated into nude mice. Twenty-seven days after the injection, the volumes of those tumors formed in the A375-BANCR KD group were substantially smaller than those in the control group (A375-GL2) (Fig. $1 \mathrm{~g})$. However, the volumes of those tumors formed in the A375-BANCR OE group were substantially bigger relative to control A375-LV5 group (Fig. 1h). Furthermore, the mean tumor weight in the A375BANCR KD group at the end of the experiment was significantly lower compared with the control group (Fig. 1i). By contrast, the mean tumor weight in the A375-BANCR OE group was markedly higher than the control group (Fig. 1j). All above results suggested that LncRNA BANCR promoted melanoma cells growth in vivo.

\section{LncRNA BANCR promotes cancer cell migration and invasion in vitro}

By transwell assay, we showed that knockdown of BANCR inhibited A375 cells migration and invasion, whereas overexpression of BANCR promoted A375 cells migration and invasion (Fig. 2a, b). Several studies have shown that increased expression of MMP2 and MMP9 induced cancer cell migration and invasion. Therefore, we next tested whether MMP2 and MMP9 were involved in BANCR-driven melanoma cells invasion and migration. We treated A375-BANCR OE and A375-BANCR KD cells with SB-3CT, an effective competitive metalloproteinase MMP-2 and MMP-9 inhibitor, and found that SB-3CT significantly inhibited the migration and invasion ability of A375-BANCR OE and A375BANCR KD cells (Fig. 2C). In addition, forced expression of BANCR in A375 cells significantly increased MMP2 and MMP9 protein expression, and knockdown of BANCR remarkably decreased MMP2 and MMP9 protein expression (Fig. $2 \mathrm{~d}$ ). These results suggested that LncRNA BANCR promoted the migration and invasion of melanoma cells by upregulating MMP2 and MMP9 protein expression.

\section{LncRNA BANCR promotes metastasis of melanoma cells in vivo}

Enhanced cell migration and invasion is essential for cancer cells metastasis in vivo. Tail vein assay data revealed that BANCR overexpression markedly increased lung colonization, whereas knockdown of BANCR strongly decreased lung colonization (Fig. 3a, b). All these results were further confirmed by HE and IHC staining (Fig. 3c).

\section{LncRNA BANCR promotes cancer cell growth and migration by activating glucose metabolism pathway}

We next assessed whether BANCR showed any effect on glucose metabolism in melanoma cells. Therefore, we detected the PFK activity and PFK1 mRNA levels in A375-BANCR KD and A375-BANCR OE cells. As shown in Fig. S1a, knockdown of BANCR decreased the PFK activity, whereas overexpression of 
BANCR increased the PFK activity in A375 cells. Consistant with these results, the mRNA levels of PFK1 were decreased in A375-BANCR KD cells relative to control cells, whereas A375-BANCR OE cells exerted higher PFK1 mRNA levels than A375-WT cells (Fig. S1b). Additionally, pyruvate kinase activities were upregulated by BANCR in A375 cells, whereas knockdown of BANCR inhibited the pyruvate kinase activities (Fig. S1c). Moreover, we detected the PKM2 mRNA levels in A375-BANCR KD and A375-BANCR OE cells. As shown in Fig. S1d, PKM2 mRNA levels were upregulated by BANCR overexpression and attenuated by BANCR knockdown in A375 cells.

Pentose phosphate pathway is an important glucose metabolism pathway, which has a fundamental role in tumor growth and migration [20]. Therefore, we detected the activities, mRNA and protein levels of glucose-6-phosphate dehydrogenase (G6PD), a key enzyme of pentose phosphate pathway. We showed that the activities, mRNA and protein levels of G6PD were also upregulated by BANCR overexpression and attenuated by BANCR knockdown in A375 cells (Fig. 4a, b and c). G6PD mRNA levels were also analyzed in 48 malignant human melanoma sample and adjacent normal tissue. As shown in Fig. $4 \mathrm{~d}$, the gene mRNA levels were significantly upregulated in melanoma tissues relative to adjacent normal tissues.

\section{LncRNA BANCR promotes G6PD expression by suppressing miR-206 and miR-571 expression}

LncRNA usually acts as a miRNA sponge to play important roles in regulating tumor formation. Therefore, we wondered whether LncRNA BANCR promoted G6PD expression by suppressing miRNA expression. LncRNA BANCR-targeted miRNAs were screened using an online prediction software Microlnspector (http://bioinfo.uni-plovdiv.bg/microinspector/). Additionally, these miRNAs targeting G6PD were predicted using online software miRwalk 3.0 (http://mirwalk.umm.uni-heidelberg.de/). We found 16 predicted BANCR-targeted miRNAs which were potentially involved in regulating G6PD expression. Therefore, we detected these miRNA levels in overexpressed BANCR and knockdown of BANCR A375 cells. As shown in Fig. 5a, miR-206 and miR-571 were markedly suppressed by BANCR overexpression, whereas the two miRNAs were significantly increased by BANCR knockdown in A375 cells. Moreover, A375 cells were transiently transfected with BANCR overexpression vector or BANCR siRNA. And then miR-206 and miR-571 levels were analysed by RT-PCR. We found that miR-206 and miR571 levels were suppressed in A375 cells transiently transfected with BANCR vector, whereas the both miRNAs were upregulated by BANCR siRNA (Fig. $5 b$ ). The results were consistent with the data from A375-BANCR KD and A375-BANCR OE cells.

To detect whether the levels of miR-206 and miR-571 were lower in melanoma cells than normal cells, we examined the two miRNAs expression in five melanoma cell lines A375, A875, sk-mel-110, M21 and M28 cells, and a transformed aneuploid immortal keratinocyte Hacat cell line. As shown in Fig. $5 c$, the levels of miR-206 and miR-571 were obviously higher than the five melanoma cell lines.

Above results showed IncRNA BANCR promoted G6PD expression in A375 cells. To evaluate the role of miR-206 and miR-571 in G6PD expression induced by BANCR, we ftransfected the A375 cells using mimics or inhibitor of the two miRNAs. Our results showed that both miR-206 and miR-571 mimics significantly attenuated mRNA levels of G6PD, whereas both miR-206 and miR-571 inhibitors observably 
enhanced the mRNA levels of the G6PD gene in A375 cells (Fig. 5d). Moreover, we detected the G6PD activity in A375 cells transfected with the two miRNAs mimics and inhibitor. Similarly, we found that miR206 and miR-571 mimics significantly inhibited G6PD activity, whereas both miRNA inhibitors observably enhanced G6PD activity in A375 cells (Fig. 5e).

The potential binding sites of miR-206 and miR-571 were shown in Fig. $5 f$ and Fig. S2. The wild-type and mutated BANCR were cloned into the downstream of firefly luciferase coding region in pGL3 reporter plasmid respectively (Fig. 5f). The vectors were co-transfected with mimics or inhibitors of miR-206 and miR-571 into A375 cells, respectively. As shown in Fig. 5f, the miR-571 mimics significantly inhibited luciferase activity, whereas miR-571 inhibitor increased the activity in A375 cells transfected wild-type reporter vectors. However, both miR-206 mimics and inhibitors failed to change the luciferase activity of wild type BANCR reporter (BANCR WT) (Fig. S2b) and mutant BANCR reporter (BANCR MUT) (Fig. S2C). The potential binding site of miR-206 on 3'UTR of BANCR was displayed in Fig. S2a. Thess results suggested only miR-571 directly binded to BANCR, and miR-206 failed to bind the BANCR. BANCR directly bound to and downregulated miR- 571 levels. The miR-206 was indirectly suppressed by BANCR.

\section{MiR-206 and miR-571 inhibit G6PD expression by directly binding the 3'UTR of G6PD}

To further confirm targeting of BANCR by miR-206 and miR-571, we predicted the potential binding sites of the two miRNAs in 3'UTR of G6PD (Fig. 6a, d). Luciferase activity assay was used to validate the potential binding sites. As shown in Fig. $6 \mathrm{~b}$ and e, miR-206 and miR-571 mimics significantly decreased luciferase activity in A375 cells transfected wild type (WT) reporter vectors. Contrarily, in A375 cells cotransfected with miR-206 or miR-571 inhibitors as well as WT reporter vectors, the luciferase activities were higher than that in cells treated with negative control (NC) miRNA inhibitor (Fig. 6b, e). However, no obvious change of luciferase activities by mimics or inhibitor of miR-206 and miR-571 was observed in A375 cells transfected with mutant type (MT) reporter vectors (Fig. 6c, f).

\section{MiR-206 and miR-571 inhibited the proliferation of melanoma cells induced by BANCR}

To investigate the roles of miR-206 and miR-571 in the growth of melanoma cells, A375 cells were treated with miR-206 or miR-571 mimics, and the cell viability was examined. As shown in Fig. 7a and Fig. 7c, forced expression of miR-206 or miR-571 mimics in A375 and A375-BANCR OE cells significantly decreased cell viability. By contrast, both miRNA inhibitors significantly increased the cell viability in A375 (Fig. 7a) and A375-BANCR KD cells (Fig. 7b). We treated A375-BANCR OE cells using mimics of miR-206 and miR-571. As shown in Fig. 7c, miR-206 and miR-571 mimics apparently decreased cell viability. By colony formation assay, we performed the rescue experiment. We showed that forced expression of miR206 or miR-571 using mimics in A375 cells significantly decreased cell colony formation increased by BANCR overexpression (Fig. 7d, e). On the contrary, depletion of miR-206 or miR-571 using miRNA inhibitors significantly increased the cell colony formation decreased by BANCR knockdown in A375 cells (Fig. 7d, e). These results suggested that MiR-206 and miR-571 inhibited the proliferation of melanoma cells induced by BANCR. 


\section{Discussion}

MMPs are capable of degrading most of extracellular matrix proteins to promote cell migration [17, 21]. Here, our results suggested that BANCR enhanced melanoma cells migration was involved in MMP2 and MMP9. The attenuation of BANCR by shRNA markedly increased the ratio of cells population in G1 phase. Moreover, this block effect of G1 phase appeared to be linked in the repression of cyclinD1 and CDK2. In addition, we detected the protein expression of p27, an inhibitor of cyclin-CDK protein kinase activity. Our results showed BANCR did not change the p27 expression in A375 cells (data not shown). Furthermore, induction of tumor growth and migration effects of BANCR appeared to be associated with glucose metabolism pathway. Lastly, we showed BANCR acted as a ceRNA of miR-571 to induce G6PD expression in melanoma. It has become largely accepted that IncRNAs can function as miRNA sponges and compete for miRNA binding to regulate mRNAs levels [22]. For example, linc-MD1 can act as a sponge to block miR-133 from binding to MAML1 and MEF2C during muscle differentiation [23]. Additionally, in liver cancer, IncRNA HULC acts as a decoy to suppress miR-372, thereby resulting in abolition of the translational repression of miR-372 target gene PRKACB [24]. Furthermore, IncRNA BC032469 as a novel ceRNA sequesters miR-1207-5p and therefore upregulates hTERT expression in gastric cancer [25]. Overall, these results were consistant with our data that LncRNA might function as ceRNA to regulate gene expression by inhibiting miRNA. However, miR-206 expression was regulated indirectly by BANCR. The mechanism of miR-206 downregulated by BANCR was unclear. Several studies have reported that long non-coding RNA MIR4435-2HG, SNHG14 and LINC00707 can directly bind miR206 to suppress it expression [26-28]. Therefore, we speculated BANCR decreased the miR-206 expression was involved in regulating these LncRNA expression. This mechanism will be the focus of our next study.

In this study, we showed miR-206 and miR-571 exerted anti-tumor effects to inhibit A375 cells proliferation. Previous study also showed that miR-206 inhibits cell proliferation by repressing cyclinD2 in gastric cancer [29]. In breast cancer, miR-206 inhibits cell stemness and migration by targeting MKL1/IL11 pathway [30]. Recently, Adams et al found that miR-206 directly binds the 3'-untranslated region (UTR) of the ERa transcript and decreases endogenous ERa mRNA and protein levels in human breast cancer cells [31, 32]. MiR-571 is deregulated in human serum in liver cirrhosis [33]. However, its role in cancer is unclear. Here, we firstly found miR-571 exerted anti-tumor role in melanoma cells.

Aerobic glycolysis is the most prominent metabolic feature in cancer cells. This phenomenon is known historically as the Warburg effect [21]. Warburg effect induces a large-scale biosynthetic program to promote cancer cell proliferation and tumor development [34-36]. We showed BANCR induced the upregulation of phosphofructokinase, pyruvate kinase and G6PD, the enzyme associated with aerobic glycolysis. These results implied that BANCR promoted aerobic glycolysis in melanoma cancer cells. G6PD functions as a key enzyme to produce NADPH which maintains reduced glutathione (GSH) [20]. GSH can protect cancer cell from oxidative damage by scavenging reactive oxygen species (ROS) [37]. Previous studies indicated that G6PD strongly promotes cancer cell proliferation in vitro and in vivo [38]. Moreover, G6PD is overexpressed in many cancer types and positively correlated with poor prognosis, whereas tumor patients harboring G6PD inactivation exerted longer survival $[23,39,40]$. Here, we also 
found that G6PD was high expressed in tumor relative to normal tissues. These results suggested G6PD overexpression was involved in induction of tumor growth and migration in melanoma.

\section{Conclusion}

In summary, our study was the first to show that BANCR was overexpressed in melanoma tissues compared with corresponding adjacent normal tissues. Furthermore, it showed that high expression of BANCR promoted melanoma cell proliferation and migration in vitro and in vivo. Moreover, BANCR facilitated cell proliferation and migration was involved in attenuating levels of miR-206 and miR-571. These results indicated that BANCR acted as an oncogene in melanoma and was a potential predictive biomarker and therapy target for melanoma.

\section{Abbreviations}

LncRNAs: Long noncoding RNAs; MMPs: matrix metalloproteinases; MREs: miRNA response elements; ceRNA: competing endogenous RNA; BANCR: BRAF-activated non-protein coding RNA; EMT: epithelialmesenchymal transition; PFK: phosphofructokinase; MT: mutant type; 3'UTR 3'-untranslated region; ROS: reactive oxygen species.

\section{Declarations}

\section{Ethics approval and consent to participate}

The study was conducted in accordance with the Declaration of Helsinki principles. It was approved by the Medical Research Ethics Committee of Kunming Medical University. Written informed consent was obtained from all participants included in this study according to the committee regulations.

\section{Consent for publication}

Not applicable.

\section{Availability of data and materials}

The data used or analyzed during this study were included in this article.

\section{Competing interests}

The authors declare that they have no competing interests.

\section{Funding}

This study was supported by the National Natural Science Foundation of China (grant nos. 81460421, 31660246 and 81760455) and the Yunnan Province and Kunming Medical University of Applied Basic 
Research Joint Special Funds for Major Projects (grant nos. 2018FE468(-001)®2017FE468(-003) and 2017FE468(-072)).

\section{Authors' Contributions}

$Y Z, Y K$ conceived and designed the study. YY, HY, ZY, LY, YN, MZ, GX performed the experiments and analyzed the data. YLA, ZY, QZ assist the experiments. YY, HY, YZ, YK performed manuscript. All authors read and approved the final manuscript.

\section{Acknowledgements}

Not applicable.

\section{References}

1. Jemal A, Bray F, Center MM, et al. Global cancer statistics. CA: a cancer journal for clinicians. 2011; 61(2):69-90.

2. New Engl, J. Melanoma research gathers momentum, Lancet. 2015; 385:2323.

3. Cesana $M$, Cacchiarelli $D$, Legnini I, et al. A long noncoding RNA controls muscle differentiation by functioning as a competing endogenous RNA. Cell. 2011; 147(2):358-369.

4. Atianand MK, Hu W, Satpathy AT, et al. A Long Noncoding RNA lincRNA-EPS Acts as a Transcriptional Brake to Restrain Inflammation. Cell. 2016; 165(7):1672-1685.

5. Gomez-Maldonado L, Tiana M, Roche 0 , et al. EFNA3 long noncoding RNAs induced by hypoxia promote metastatic dissemination. Oncogene. 2015; 34(20):2609-2620.

6. Heilmann K, Toth R, Bossmann C, et al. Genome-wide screen for differentially methylated long noncoding RNAs identifies Esrp2 and IncRNA Esrp2-as regulated by enhancer DNA methylation with prognostic relevance for human breast cancer. Oncogene. 2017; 36(46):6446-6461.

7. Huang $S$, Lu W, Ge D, et al. A new microRNA signal pathway regulated by long noncoding RNA TGFB2-OT1 in autophagy and inflammation of vascular endothelial cells. Autophagy. 2015; 11(12):2172-2183.

8. Wang D, Wang D, Wang N, et al. Long Non-Coding RNA BANCR Promotes Endometrial Cancer Cell Proliferation and Invasion by Regulating MMP2 and MMP1 via ERK/MAPK Signaling Pathway. Cell Physiol Biochem. 2016; 40(23):644-656.

9. Li J, Wang J, Zhou W, et al. Downregulation of BRAF-activated non-coding RNA suppresses the proliferation, migration and invasion, and induces apoptosis of hepatocellular carcinoma cells. Oncol Lett. 2017; 14(4):4751-4757.

10. Zhang ZX, Liu ZQ, Jiang B, et al. BRAF activated non-coding RNA (BANCR) promoting gastric cancer cells proliferation via regulation of NF-kappaB1. Biochem Biophys Res Commun. 2015; 465(2):225- 
231.

11. Zheng $H$, Wang $M$, Jiang $L$, et al. BRAF-Activated Long Noncoding RNA Modulates Papillary Thyroid Carcinoma Cell Proliferation through Regulating Thyroid Stimulating Hormone Receptor. Cancer Res Treat. 2016; 48(2):698-707.

12. Hu L, Li M, Pu L, et al. The different prognostic value of long non-coding RNA BANCR in human cancers. Minerva Med. 2017; 108(1):97-100.

13. Sun M, Liu XH, Wang KM, et al. Downregulation of BRAF activated non-coding RNA is associated with poor prognosis for non-small cell lung cancer and promotes metastasis by affecting epithelialmesenchymal transition. Mol Cancer. 2014; 13(1):68.

14. Zou Z, Yuan Z, Zhang Q, et al. Aurora kinase A inhibition-induced autophagy triggers drug resistance in breast cancer cells. Autophagy. 2012; 8(12):1798-1810.

15. Ao X, Nie P, Wu B, et al. Decreased expression of microRNA-17 and microRNA-20b promotes breast cancer resistance to taxol therapy by upregulation of NCOA3. Cell Death Dis. 2016; 7(11):2463.

16. Nie P, Hu W, Zhang T, et al. Synergistic Induction of Erlotinib-Mediated Apoptosis by Resveratrol in Human Non-Small-Cell Lung Cancer Cells by Down-Regulating Survivin and Up-Regulating PUMA. Cell Physiol Biochem. 2015; 35(6):2255-2271.

17. Luo X, Yao J, Nie P, et al. FOXM1 promotes invasion and migration of colorectal cancer cells partially dependent on HSPA5 transactivation. Oncotarget. 2016; 7(18):26480-26495.

18. Chen P, Luo X, Nie P, et al. CQ synergistically sensitizes human colorectal cancer cells to SN-38/CPT11 through lysosomal and mitochondrial apoptotic pathway via p53-R0S cross-talk. Free Radic Biol Med. 2017; 104(1):280-297.

19. Wang S, Zou Z, Luo X, et al. LRH1 enhances cell resistance to chemotherapy by transcriptionally activating MDC1 expression and attenuating DNA damage in human breast cancer. Oncogene. 2018; 37(24):3243-3259.

20. Mele L, Paino F, Papaccio F, et al. A new inhibitor of glucose-6-phosphate dehydrogenase blocks pentose phosphate pathway and suppresses malignant proliferation and metastasis in vivo. Cell Death Dis. 2018; 9(5):1-12.

21. Hanahan D, Weinberg RA. Hallmarks of cancer: the next generation. Cell 144(5):646-674.

22. Salmena L, Poliseno L, Tay Y, et al. A ceRNA hypothesis: the Rosetta Stone of a hidden RNA language? Cell. 2011; 146(3):353-358.

23. Legnini I, Morlando M, Mangiavacchi A, et al. A feedforward regulatory loop between HuR and the long noncoding RNA linc-MD1 controls early phases of myogenesis. Mol Cell. 2014; 53(3):506-514.

24. Wang J, Liu X, Wu H, et al. CREB up-regulates long non-coding RNA, HULC expression through interaction with microRNA-372 in liver cancer. Nucleic Acids Res. 2010; 38(16):5366-5383.

25. Lu MH, Tang B, Zeng S, et al. Long noncoding RNA BC032469, a novel competing endogenous RNA, upregulates hTERT expression by sponging miR-1207-5p and promotes proliferation in gastric cancer. Oncogene. 2016; 35(27):3524-3534. 
26. Jiang X, Liu Y, Zhang G, et al. Acetyl-11-Keto-B-Boswellic Acid Inhibits Precancerous Breast Lesion Mcf-10at Cells Via Regulation of Linc00707/Mir-206 That Reduces Estrogen Receptor-A. Cancer management and research. 2020; 12(1):2301-2314.

27. Dong X, Yang Z, Yang H, et al. Long Non-Coding Rna Mir4435-2hg Promotes Colorectal Cancer Proliferation and Metastasis through Mir-206/Yap1 Axis. Frontiers in oncology. 2020; 10: 160-173.

28. Zhao L, Zhang X, Shi Y, et al. LncRNA SNHG14 contributes to the progression of NSCLC through miR206/G6PD pathway. Thorac Cancer. 2020; 11(5).

29. Zhang L, Liu X, Jin H, et al. miR-206 inhibits gastric cancer proliferation in part by repressing cyclinD2. Cancer Lett. 2013; 332(1):94-101.

30. Samaeekia R, Adorno-Cruz V, Bockhorn J, et al. miR-206 Inhibits Stemness and Metastasis of Breast Cancer by Targeting MKL1/IL11 Pathway. Clin Cancer Res. 2017; 23(4):1091-1103.

31. Adams BD, Cowee DM, White BA. The role of miR-206 in the epidermal growth factor (EGF) induced repression of estrogen receptor-alpha (ERalpha) signaling and a luminal phenotype in MCF-7 breast cancer cells. Mol Endocrinol. 2009; 23(8):1215-1230.

32. Zhu J, Zou Z, Nie P, et al. Downregulation of microRNA-27b-3p enhances tamoxifen resistance in breast cancer by increasing NR5A2 and CREB1 expression. Cell Death Dis. 2016; 7(11):2454-2454.

33. Roderburg $C$, Mollnow T, Bongaerts $B$, et al. Micro-RNA profiling in human serum reveals compartment-specific roles of miR-571 and miR-652 in liver cirrhosis. Plos One. 2012; 7(3):e32999.

34. Mi YJ, Geng GJ, Zou ZZ, et al. Dihydroartemisinin inhibits glucose uptake and cooperates with glycolysis inhibitor to induce apoptosis in non-small cell lung carcinoma cells. Plos One. 2015; 10(5):e120426.

35. Jiang S, Zou Z, Nie P, et al. Synergistic Effects between mTOR Complex $1 / 2$ and Glycolysis Inhibitors in Non-Small-Cell Lung Carcinoma Cells. Plos One. 2015; 10(7):e132880.

36. Lou YF, Zou ZZ, Chen PJ, et al. Combination of gefitinib and DNA methylation inhibitor decitabine exerts synergistic anti-cancer activity in colon cancer cells. Plos One. 2014; 9(5):e97719.

37. Zou Z, Chang H, Li H, et al. Induction of reactive oxygen species: an emerging approach for cancer therapy. Apoptosis. 2017; 22(11):1321-1335.

38. Du W, Jiang P, Mancuso A, et al. TAp73 enhances the pentose phosphate pathway and supports cell proliferation. Nat Cell Biol. 2013; 15(8):991-1000.

39. Compagno M, Lim WK, Grunn A, et al. Mutations of multiple genes cause deregulation of NF-kappaB in diffuse large B-cell lymphoma. Nature. 2009; 459(7247):717-721.

40. Zhang $X$, Zhang $X$, Li Y, et al. PAK4 regulates G6PD activity by p53 degradation involving colon cancer cell growth. Cell Death Dis. 2017; 8(5):e2820.

\section{Figures}


(a)

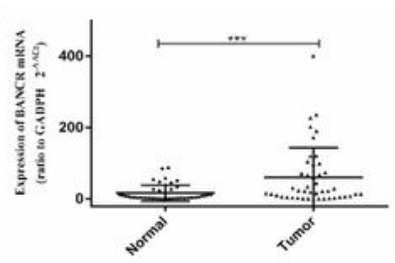

(d)

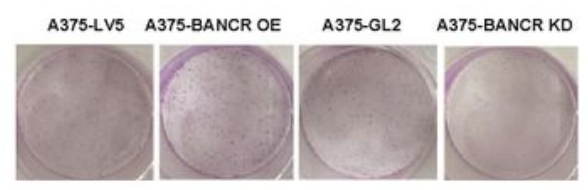

(b)

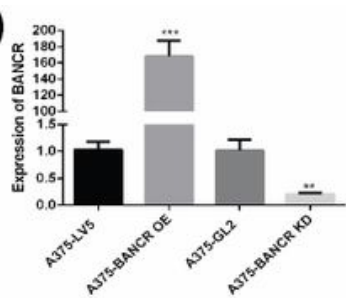

(c)
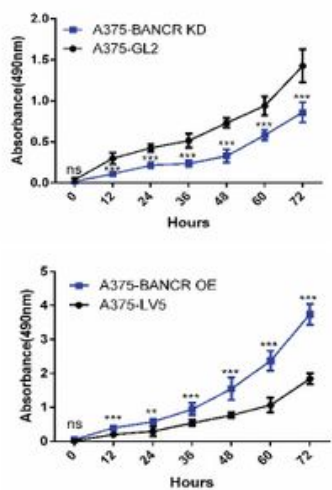

(e)


(f)
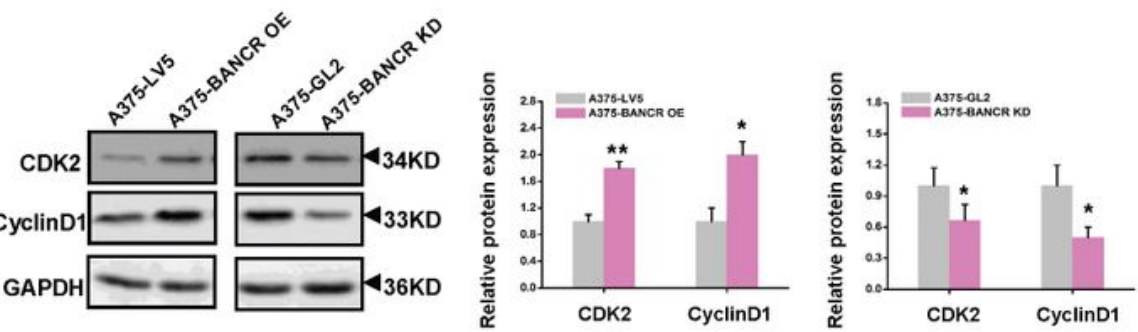

(g)

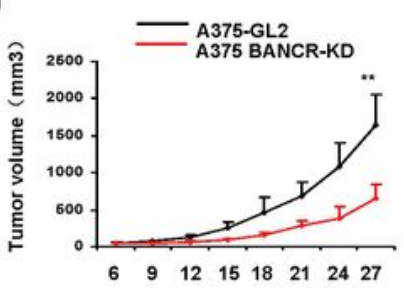

(i)



(h)

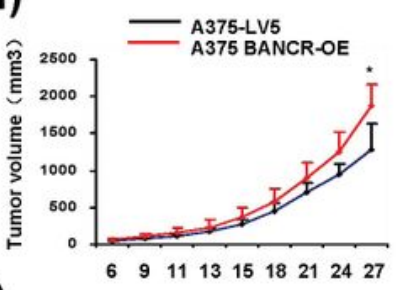

(j)

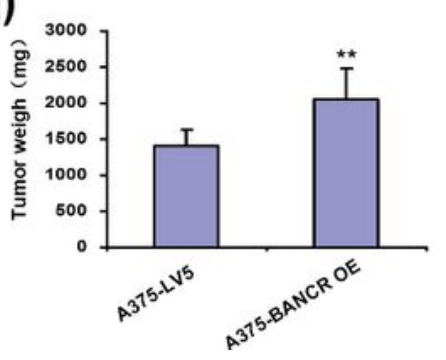

Figure 1

LncRNA BANCR is overexpressed in melanoma and promotes cell growth in vitro and in vivo a The expression levels of BANCR in 48 malignant human melanoma sample and adjacent normal tissue as measured by RT-PCR analysis. $b$ The expression levels of BANCR in stably overexpressed BANCR melanoma cells (A375-BANCR OE) and control cells A375-LV5; knockdown of BANCR cells (A375-BANCR KD) and control cells A375-GL2. The expression levels of BANCR were also determined by RT-PCR. c-e 
MTT assay (c), clonogenic cell survival analysis (d) and cell cycle analysis (e) were performed to examine cell viability in A375-BANCR OE, A375-LV5, A375-BANCR KD and A375-GL2 cells. Above experiments were repeated three times. Data represent mean \pm S.D. ${ }^{* \star} P<0.01 ;{ }^{* \star *} P<0.001$. $\mathrm{f}$ Western blot was performed to detect the protein expression of CDK2 and CyclinD1. The signals were stimulated with Enhanced Chemiluminescence Substrate and visualized on Image Quant 4000. Full-length blots are presented in Supplementary figure S3. The relative quantification of protein was analyzed by scanning densitometry using Image $\mathrm{J}$ software and GAPDH was used as a loading control. Data were from three independent experiments. g-h A375-BANCR OE, A375-LV5, A375-BANCR KD and A375-GL2 cells were injected subcutaneously into nude mice. After 33 days, these mice were sacrificed. $g$, h Cells were injected into nude mice. After 6 days, tumor volumes were measured two times a week by using calipers (as indicated at each time point) for 33 days. i, j Average tumor weight were measured. Data were shown as mean \pm S.D. $\left(n=6\right.$ per group). ${ }^{*} p<0.05,{ }^{* *} p<0.01$. 
(a)
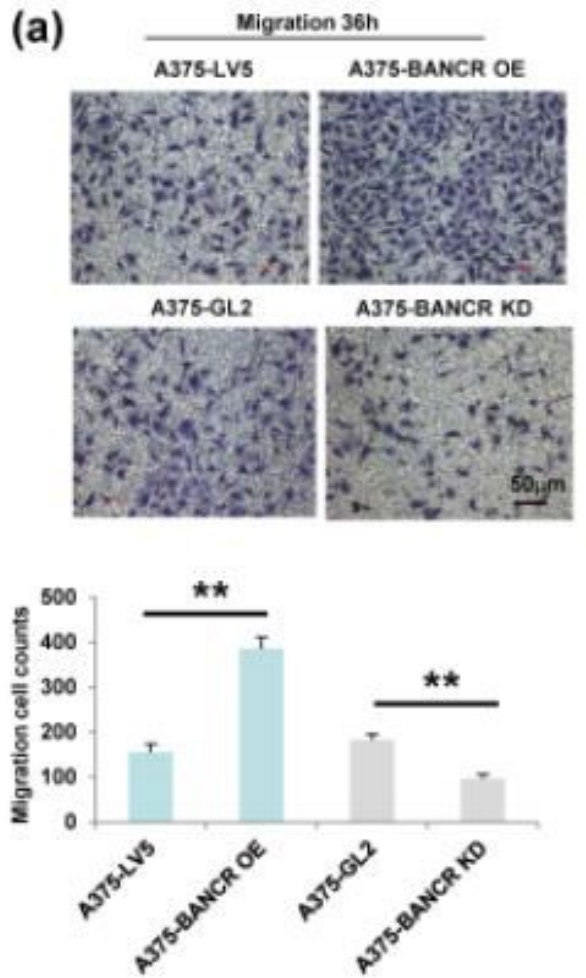

(b) $\frac{\text { Invasion 48h }}{\text { A375-LV5 A375-BANCR OE }}$
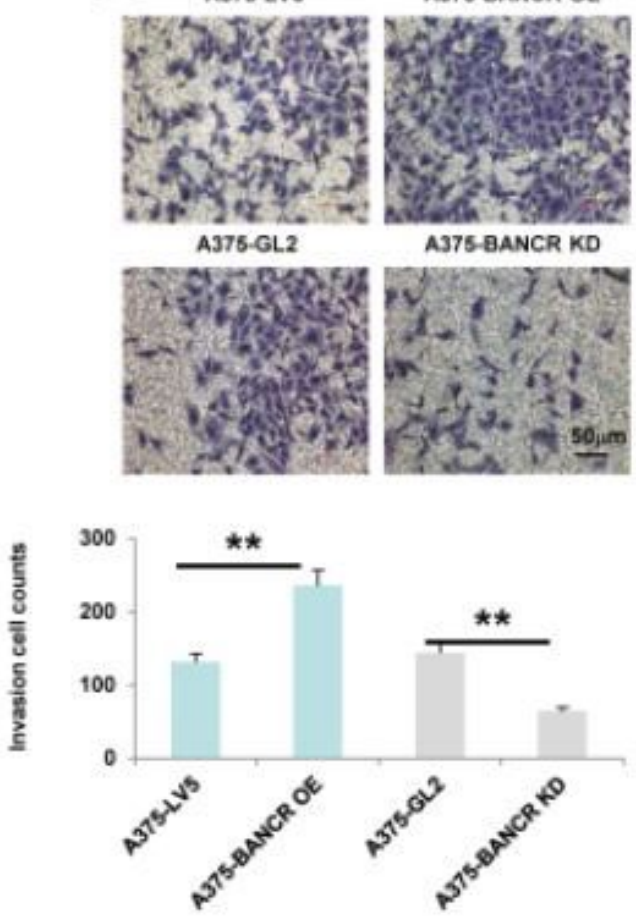

(c)
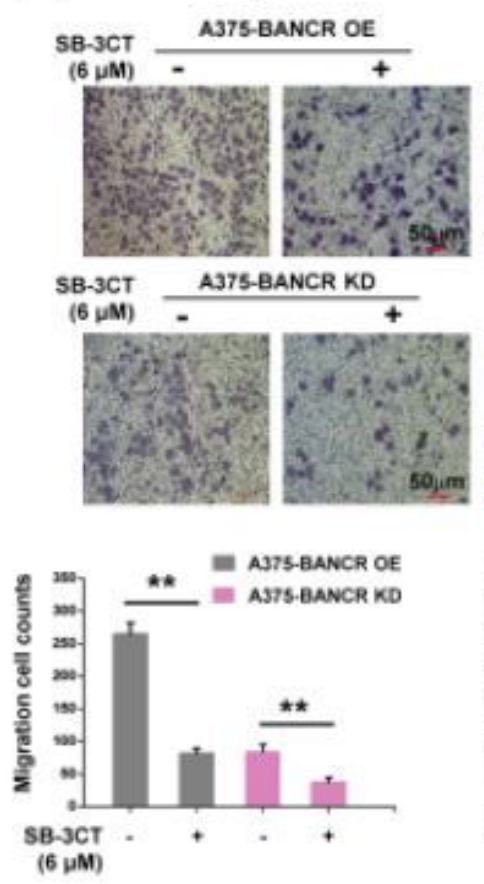
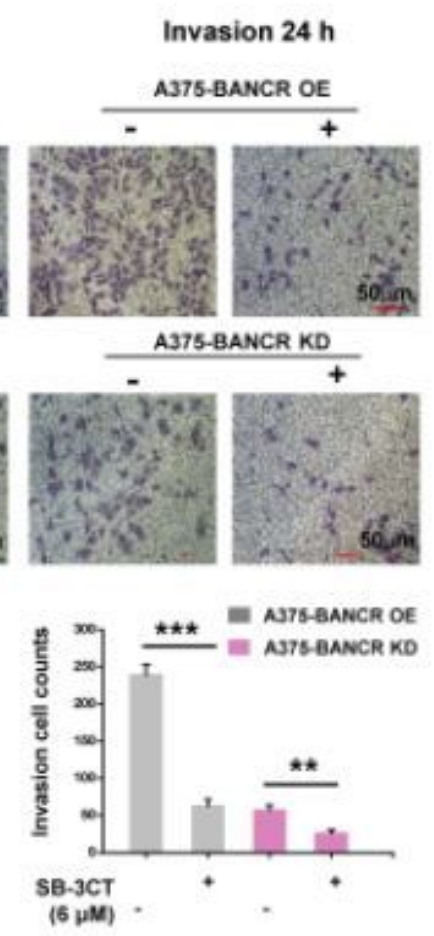

(d)

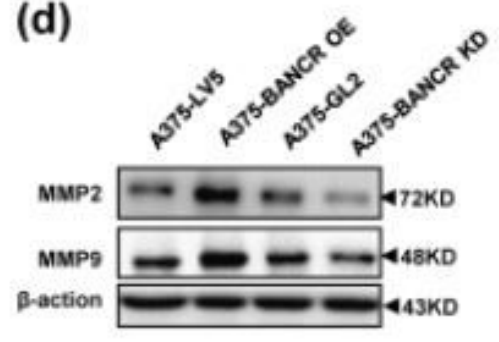

\section{Figure 2}

LncRNA BANCR promotes cancer cell migration and invasion in vitro $a, b$ Cell migration and invasion assay were performed by transwell assay in A375-BANCR OE, A375-LV5, A375-BANCR KD and A375-GL2 cells. Experiments were repeated three times. Data represent mean \pm S.D. ${ }^{*} P<0.01$. $c$ The migration and invasion ability of A375-BANCR OE and A375-BANCR KD cells were analyzed by transwell assay in the presence of SB-3CT. d Western blot was performed to detect the protein expression of MMP2 and MMP9. 
The signals were stimulated with Enhanced Chemiluminescence Substrate and visualized on Image Quant 4000. Full-length blots are presented in Supplementary figure S4. $\beta$-actin was used as a loading control. Data were from three independent experiments.

(a)

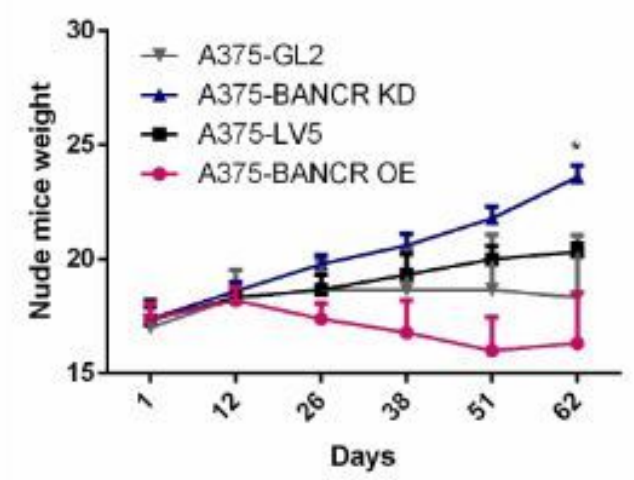

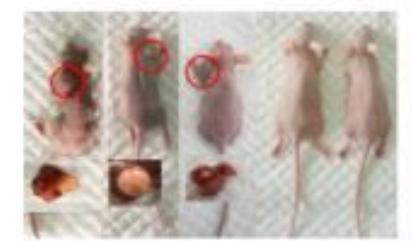

A375-BANCR OE

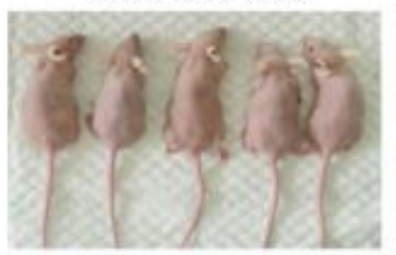

A375-BANCR KD

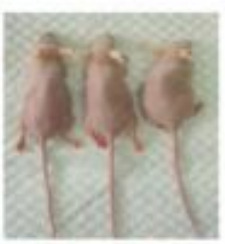

A375-LV5

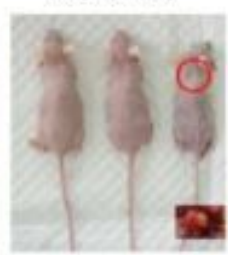

A375-GL2 (b)

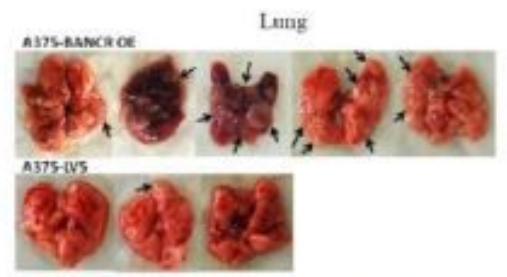

A375 BAnca 60
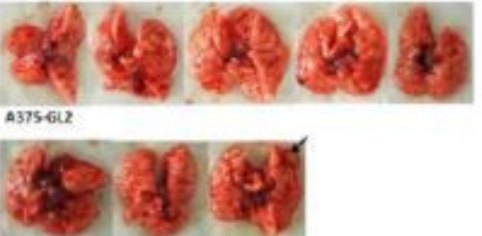

(c)
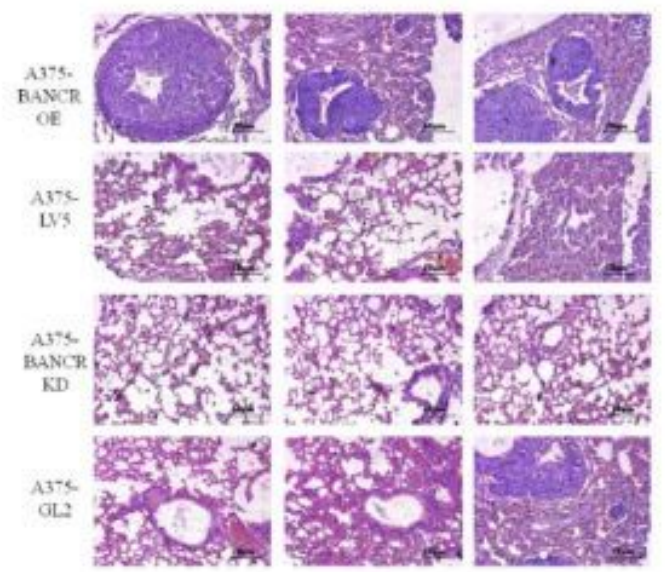

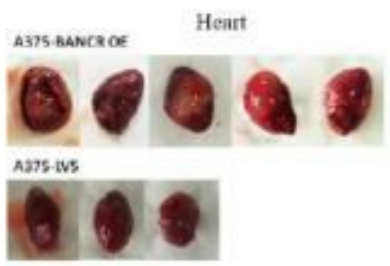

Asts-Muncako

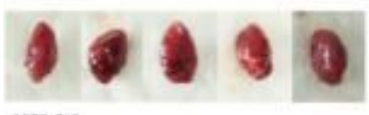

A375-64:

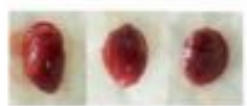

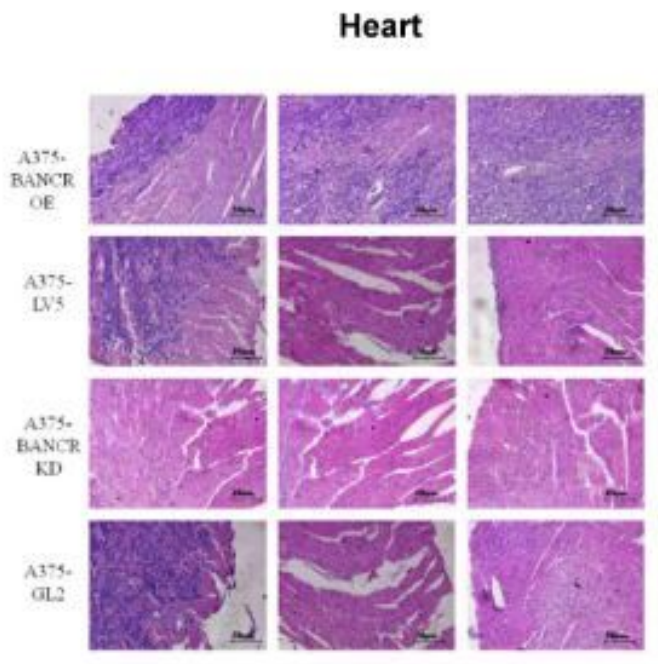

Figure 3

LncRNA BANCR promotes cancer cell metastasis in vivo a A375-BANCR OE, A375-LV5, A375-BANCR KD and A375-GL2 cells were injected intravenously into nude mice. Mice weights were measured once 12 
days. After 62 days, these mice were sacrificed. Data were shown as mean \pm S.D. ( $n=6$ per group). ${ }^{*} \mathrm{P}<0.05$. $b$ Lungs and heart from mice in each experimental group. $c$ Visualization of the entire lung and heart by HE-stained lung and heart sections (40x).

(a)

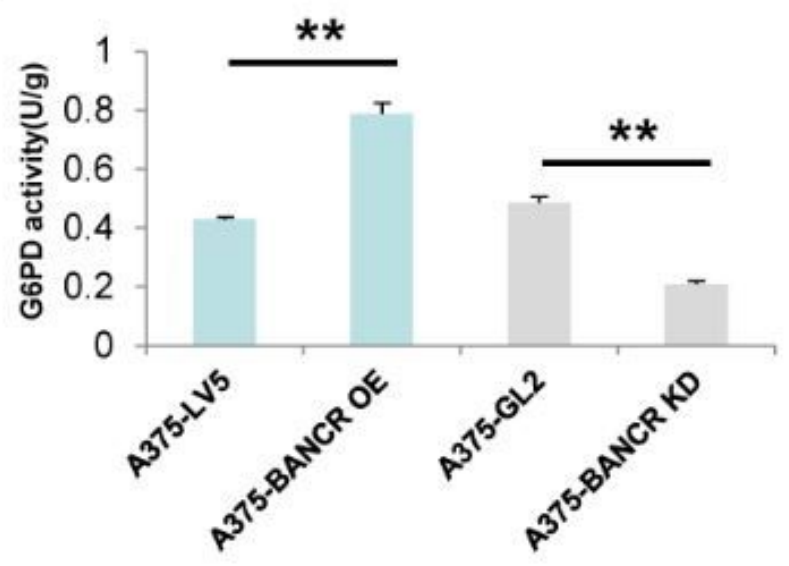

(c)

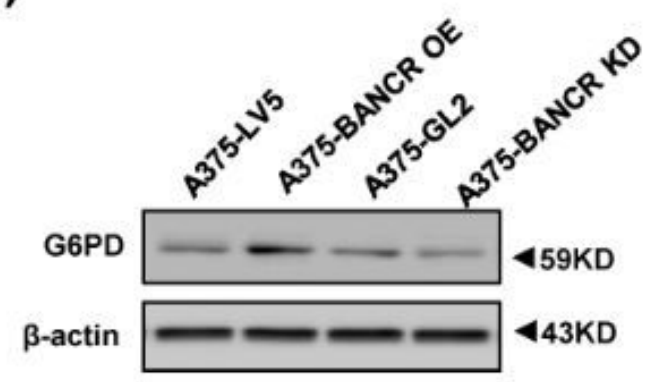

(b)



(d)

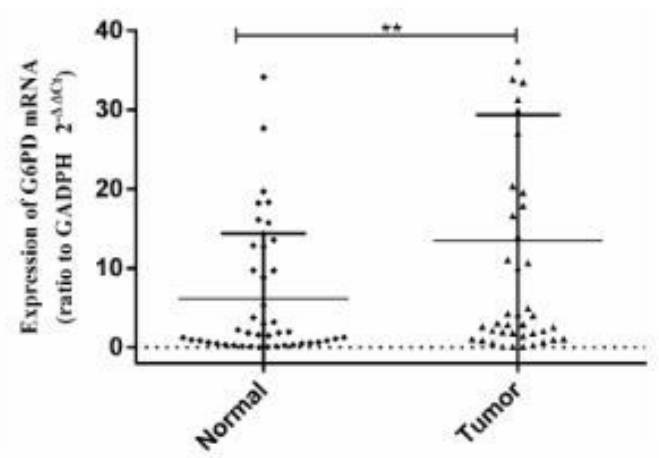

Figure 4

LncRNA BANCR upregulates G6PD expression and activities. a, b G6PD activity and mRNA levels were performed in A375-BANCR OE, A375-LV5, A375-BANCR KD and A375-GL2 cells. Experiments were repeated three times. Data represent mean \pm S.D. $* * P<0.01$. c Western blot was performed to detect the protein expression of G6PD. $\beta$-actin was used as a loading control. Data were from three independent experiments. The signals were stimulated with Enhanced Chemiluminescence Substrate and visualized on Image Quant 4000. Full-length blots are presented in Supplementary figure S5. $d$ The expression levels of G6PD in 48 malignant human melanoma sample and adjacent normal tissue as measured by RT-PCR analysis. Data represent mean \pm S.D. ${ }^{\star *} p<0.01$. 
(a)

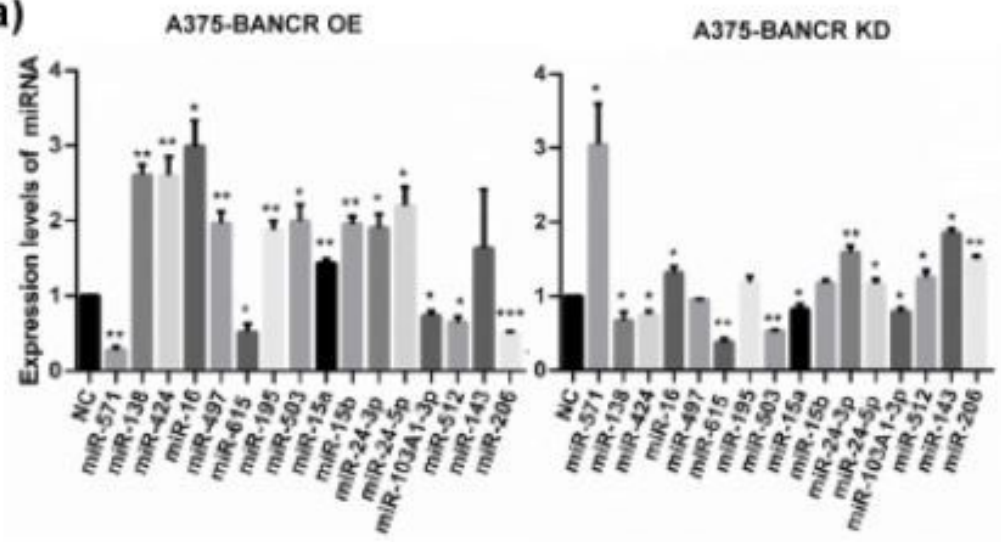

(b)

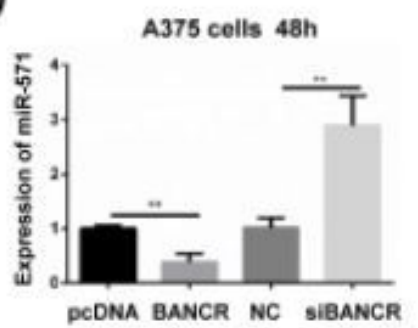

(d)

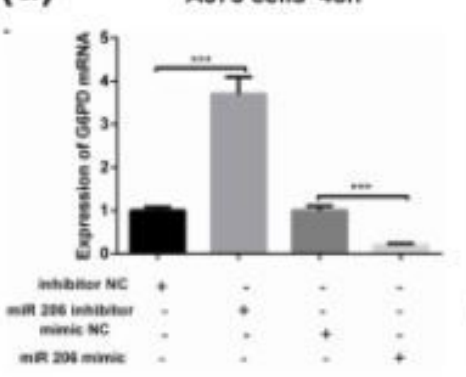

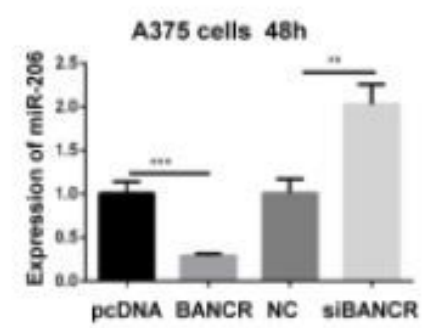



(c)
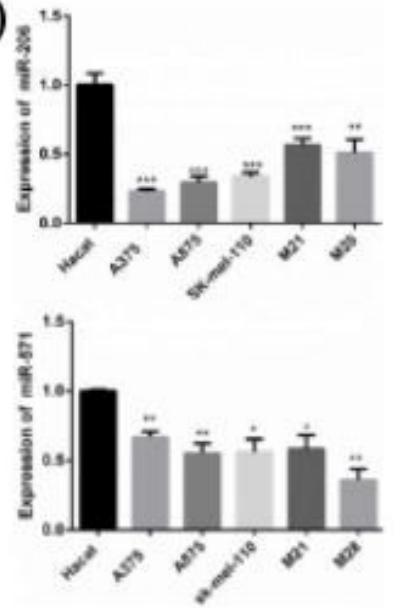

(e)
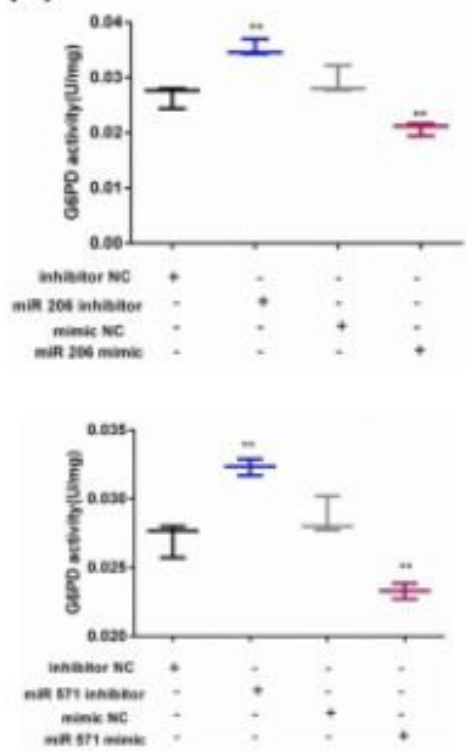

(f)

PS-CHECK2-BANCE MUT 5'ACCAC-CA-ATGGTAACCAGGTGOG 3 :

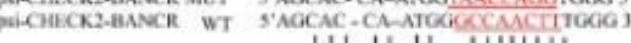
han-mik-57!

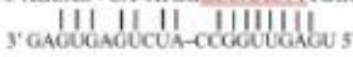


\section{Figure 5}

LncRNA BANCR promotes G6PD expression by suppressing miR-206 and miR-571 expression. a These miRNA levels in overexpressed BANCR and knockdown of BANCR A375 cells were detected by RT-PCR. NC represented the miRNA levels in control cells. b miR-206 and miR-571 expression were detected in A375 cells transiently transfected BANCR overexpression vector and BANCR siRNA by RT-PCR. c By RTPCR, miR-206 and miR-571 expression were detected in five melanoma cell lines A375, A875, sk-mel-110, 
M21 and M28 cells, and Hacat cells, a transformed aneuploid immortal keratinocyte cell line. d, e G6PD mRNA levels were detected by RT-PCR, and G6PD activities were detected in A375 cells transiently transfected mimics or inhibitors of miR-206 and miR-571. $f$ The schematic represented the predicted binding positions of putative miR- 571 on BANCR. Luciferase activity assays in A375 cells cotransfected with pGL3 vector containing the wild type (WT) or mutated (MUT) of BANCR against miR-571 bind site along with negative control (NC) or miR-571 mimics and inhibitors. After $48 \mathrm{~h}$, luciferase activity was detected. Data represent mean \pm S.D., n.s represented no significant; ${ }^{*} p<0.05,{ }^{\star \star} p<0.01,{ }^{\star \star \star} p<0.001$.

(a)

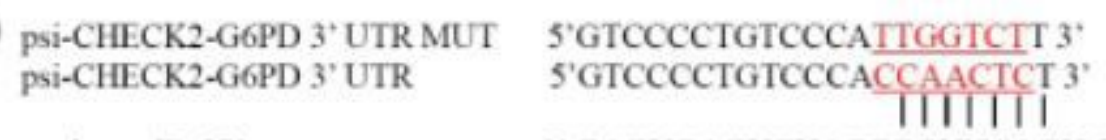

has-miR-571

3. GAGUGAGUCUACCGGUUGAGU 5 .

(b)

\section{(d)}

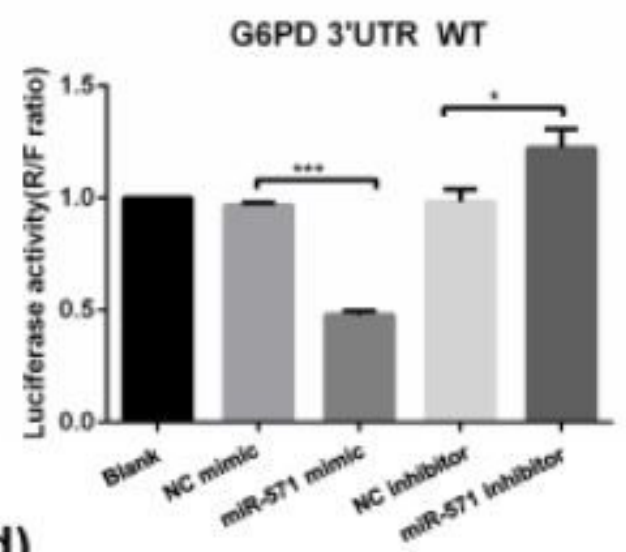

(c)

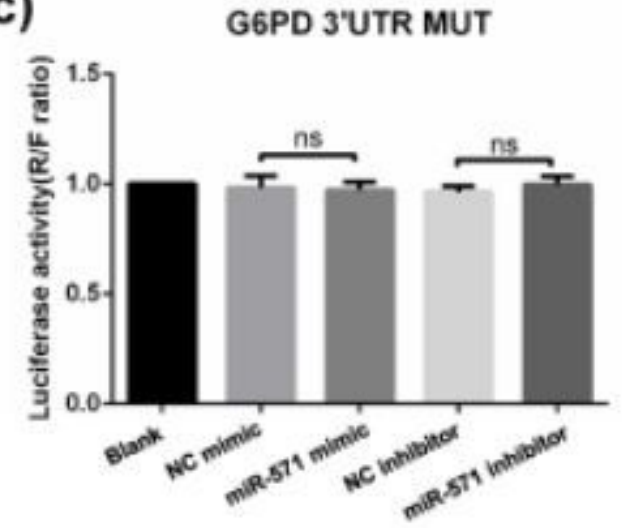

Psi-CHECK2-G6PD 3' UTR MUT psi-CHECK2-G6PD 3' UTR

has-miR-206
5.AUUGACCUCAGCUGCGUGCCUUU 3'

5'AUUGACCUCAGCUGCACAUUCCU'

\|\|\|\|

3'GGUGUGUGAAGGAAUGUAAGGU 5' (e)

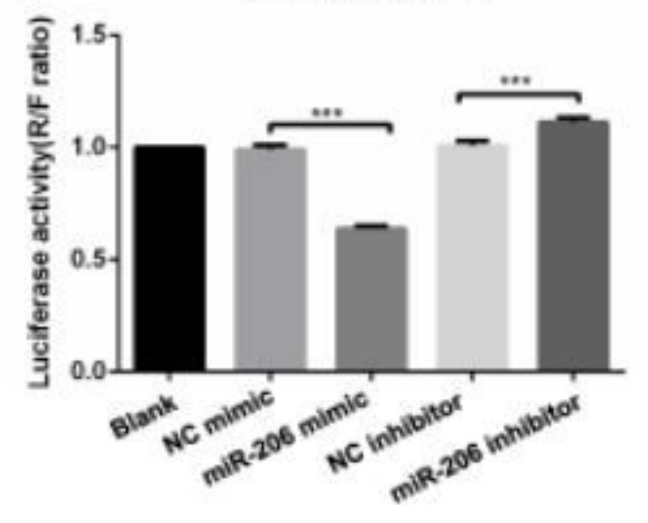

(f) G6PD 3'UTR MUT

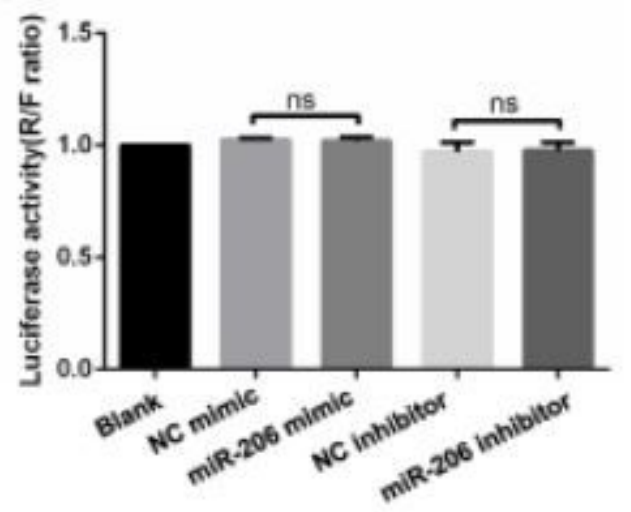

Figure 6

miR-206 and miR-571 inhibit G6PD expression by directly binding the 3'UTR of G6PD a, $d$ The schematic represented the predicted binding positions of putative miR-571 and miR-206 on G6PD 3'UTR. b-e, $f$ Luciferase activity assays in A375 cells cotransfected with pGL3 vector containing the wild type (WT) or 
mutated (MUT) 3'UTR of G6PD against miR-571 and miR-206 bind site along with negative control (NC) miR-206 or miR-571 mimics and inhibitors. After $48 \mathrm{~h}$, luciferase activity was detected. Data were normalized to luciferase activity in the corresponding cells transfected with NC and were represented as the mean \pm S.D. of three replicates. ${ }^{*} p<0.05,{ }^{* * *} p<0.001$.

(a)

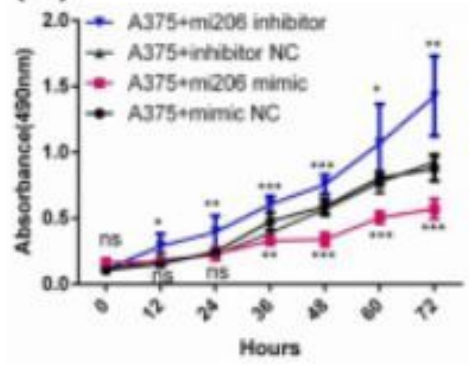

(c)

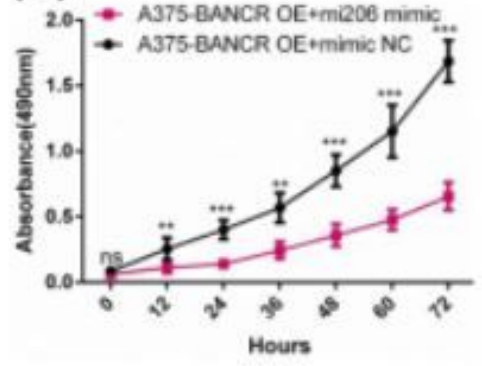

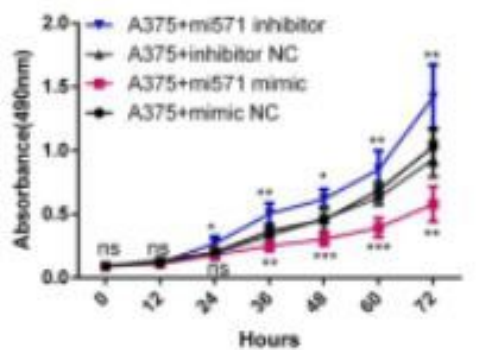

Hours (b)
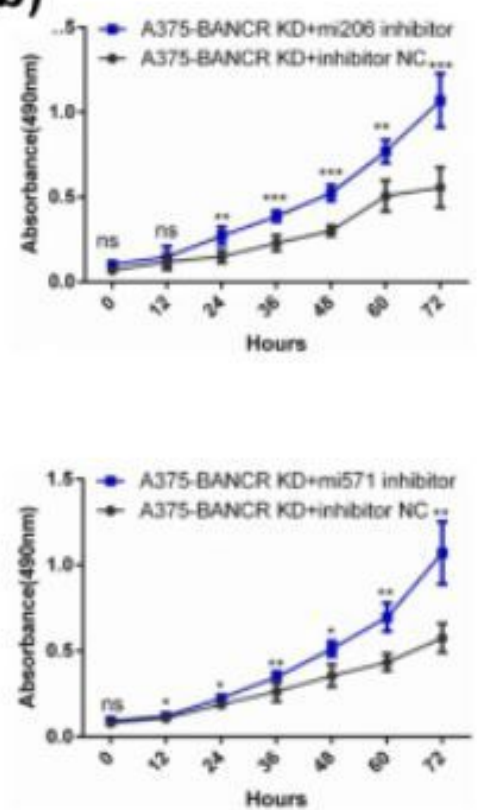

(d)

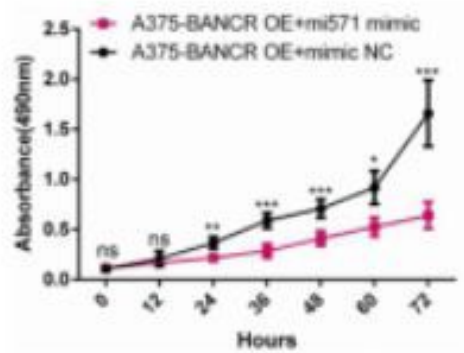

A375-BANCR KD

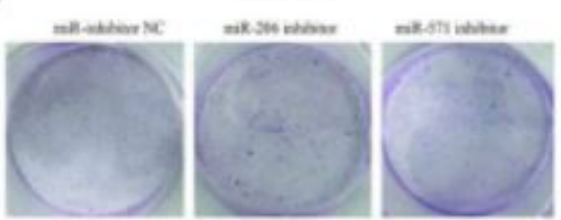

A375-GL2

mall-abes se: mak 571 wax

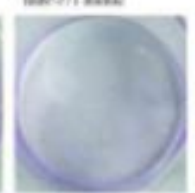

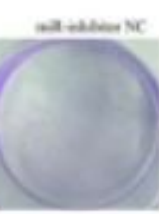

A375-BANCR OE

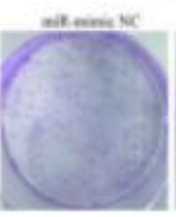
was Jat enes

(e)

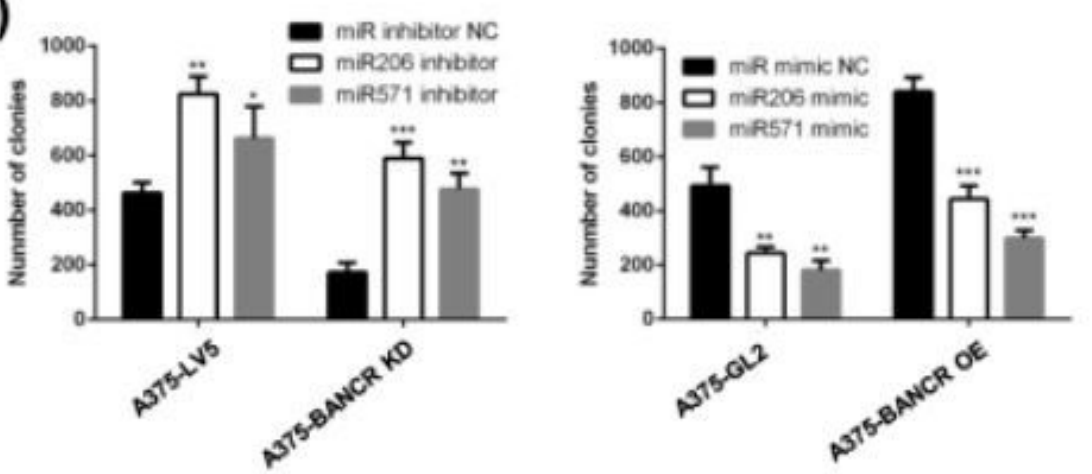

Figure 7 
miR-206 and miR-571 inhibited the proliferation of melanoma cells a-c MTT assay, d, e clonogenic cell survival analysis were performed to examine cell viability in A375, A375-BANCR OE and A375-BANCR KD cells transfected with negative control (NC), miR-206 or miR-571 mimics and inhibitors. Above experiments were repeated three times. Data represent mean \pm S.D. ${ }^{*} p<0.05 ; * \star p<0.01 ;{ }^{* \star \star} p<0.001$.

\section{Supplementary Files}

This is a list of supplementary files associated with this preprint. Click to download.

- supplementary.doc

- figS1.tif

- figS2.tif

- FigureS3.tif

- Figures4.tif

- FigureS5.tif 